\title{
Re: The Effect of Primary Surgery in Patients with De Novo Stage IV Breast Cancer with Bone Metastasis Only (Protocol BOMET MF 14-01): A Multicenter, Prospective Registry Study
}

\author{
Yumiko Ishizuka, MD, and Yoshiya Horimoto, MD, PhD \\ Department of Breast Oncology, Juntendo University School of Medicine, Bunkyo-ku, Tokyo, Japan
}

Dear Editor,

We read with interest the article by Soran et al., ${ }^{1}$ which evaluated the role of locoregional treatment (LRT) in breast cancer patients with bone-only metastasis. While the significance of LRT in stage IV breast cancer has been generally recognized as low, ${ }^{2,3}$ the authors revealed that LRT prolonged overall survival. Their data are meaningful in terms of showing the effectiveness of LRT in some populations with metastatic breast cancer. While their data are impressive, there is one aspect of this study that requires further consideration.

In their non-randomized study, some clinicopathological factors significantly differed between patients with systemic therapy alone compared with those with LRT (e.g., age, tumor size, number of bone metastasis). The LRT group was shown to have a prolonged overall survival (Fig. 1), however this univariate Kaplan-Meier analysis does not eliminate the aforementioned confounding factors. A better interpretation of these data should adjust for these factors. We believe propensity score methods should be employed, particularly for Fig. 1, as the number of participants is large enough. Re-analysis of these key data is critical to deliver more precise information to the readers.

DISCLOSURES Yumiko Ishizuka and Yoshiya Horimoto have no conflicts of interest to declare.

\section{REFERENCES}

1. Soran A, Dogan L, Isik A, Ozbas S, Trabulus DC, Demirci U, et al. The effect of primary surgery in patients with de novo stage IV breast cancer with bone metastasis only (protocol BOMET MF 14-01): a multi-center, prospective registry study. Ann Surg Oncol. 2021. https://doi.org/10.1245/s10434-021-09621-8.

2. Badwe R, Hawaldar R, Nair N, Kaushik R, Parmar V, Siddique S, et al. Locoregional treatment versus no treatment of the primary tumour in metastatic breast cancer: an open-label randomised controlled trial. Lancet Oncol. 2015;16:1380-8.

3. Khan SA, Zhao F, Solin LJ, Goldstein LJ, Cella D, Basik M, et al. A randomized phase III trial of systemic therapy plus early local therapy versus systemic therapy alone in women with de novo stage IV breast cancer: a trial of the ECOG-ACRIN Research Group (E2108). J Clin Oncol. 2020;38:LBA2.

Publisher's Note Springer Nature remains neutral with regard to jurisdictional claims in published maps and institutional affiliations.

(C) Society of Surgical Oncology 2021

First Received: 10 June 2021

Accepted: 15 June 2021;

Published Online: 12 September 2021

Y. Horimoto, MD, $\mathrm{PhD}$

e-mail: horimoto@juntendo.ac.jp 